\title{
UVA1 phototherapy as a treatment option for plaque psoriasis in HIV-positive patients
}

Cutaneous diseases are one of the most common manifestations of HIV infection and among them, psoriasis is one of the most difficult to manage.

To date, UV phototherapy represents the first-line treatment for moderate to severe psoriasis in HIV-positive patients, combined with Highly Active Antiretroviral Therapy (HAART). ${ }^{1}$

Both narrow-band UVB (NB-UVB) and photochemotherapy (PUVA) have been used as irradiation sources, based on in vitro and in vivo efficacy and safety studies. ${ }^{2}$ Here, we present the cases of five HIV-positive patients affected by plaque psoriasis, successfully treated with ultraviolet A1 (UVA1) phototherapy.

Exclusion criteria were concomitant active psoriatic arthritis, age younger than 18 years, any topical or systemic psoriasis medication (including keratolytic products) in the last 3 months before enrollment and concomitant use of systemic photosensitizing agents.

At baseline (T0) and after UVA1 phototherapy (T1) the severity of skin involvement was rated according to the Psoriasis Area and Severity Index (PASI) and physician global assessment score (PSA). At the same time points, CD4 + T-cell absolute count and percentage and HIV RNA levels were assessed.

The study was carried out in accordance with the Declaration of Helsinki and the Local Ethic Committee (protocol number 3720); all patients signed the informed consent before treatment.

UVA1 radiation was emitted by a UVA1 Dermalight UltrA1-24KW (Dr Hönle, Martinsreid, Germany) (from 1999 to 2010) and Medisun Xenia UVA1 (Schulze \& Böhm GmbH) (up to now). Irradiances were measured with a SR9910 Spectroradiometer (Macam Photometrics Ltd.) and found to be 80 and $33 \mathrm{~W} / \mathrm{m}^{2}$ at skin level, respectively.

Fixed, medium dose of $50 \mathrm{~J} / \mathrm{cm}^{2}$ UVA1 radiation was delivered 3 times weekly. This protocol was selected on the basis of results of previous studies on the treatment of psoriasis. ${ }^{3}$

Treatment was continued until complete clearance of the lesions was obtained (PASI 90) or until partial improvement (PASI 75), without further amelioration in spite of 5 additional treatments, was accomplished.

All patients were allowed to use an emollient cream as needed during treatment cycle.

Patients were followed-up with clinical examinations at intervals of 30 days for 3 months.

Continuous data were expressed as mean and range and they were analyzed using Wilcoxon matched-pairs signed rank test. Significance was defined as $P<.05$.
Pertinent clinical and treatment details are summarized in Table 1.

After treatment, PASI reduced in all patients $(P=.0003)$. (Table 1 , Figure 1).

Clinical improvement was accompanied by contrasting unchanged laboratory findings. As reported in Table 1, HIV RNA level after UVA1 phototherapy maintained stably below 50 copies $/ \mathrm{mL}$ in patients 3 and 5 while decreased in patient 1 and $4(P>.05)$ according to the starting of antiretroviral therapy. Conversely, patient 2 showed a constant positivity of HIV viremia due to a poor response to ongoing antiretroviral therapy due to a multi-drug resistant (MDR) virus.

Phototherapy induced a slight decrease in CD4 + cells percentage in three patients and a raise in two patients $(P>.05)$ (Table 1 ). No adverse events were reported with no relapses at 3-months follow-up.

NB-UVB or PUVA have been advocated as the most appropriate types of UV therapy for psoriasis in HIV-positive patients ${ }^{2}$ but data that determine which of these treatments is safer are still lacking.

Furthermore, concern about a detrimental relationship between UV and HIV infection was triggered by in vitro studies which highlighted the ability of different UV wavelengths to upregulates HIV gene expression or promote HIV replication. In order to assess whether similar effects could occur in vivo, animal studies on transgenic mice have been performed: irradiation with UVA caused no effects while UVB and UVC, natural sunlight and PUVA with 8-MOP significantly increased HIV-LTR expression. ${ }^{2}$

In these cases, lack of effect of UVA may be explained by its proper way to induce cells damage. As is known, any UV damage try to be fixed by repair enzymes inside cells; in the unwound state, cells turn on genes that may include viral genes integrated into host DNA. UVA preference to induce DNA strand breaks over pyrimidine dimers or to cause plasma membrane and cytoplasm damage over chromatin damage might justify its higher safety in the viral replication. ${ }^{4}$ Therefore, this assumption seems not to be totally exhaustive since today it is well known how UVA could act through a non-oxidative pathway too.

How could UV radiation activate or not HIV in humans needs to be still clarify. Firstly, the skin may not be a significant reservoir for HIV thus the alleged UV inducing effect on HIV replication in skin may be minimal. Moreover, the amount of UV usually delivered to the cells in vitro is greater than those provided during phototherapy 


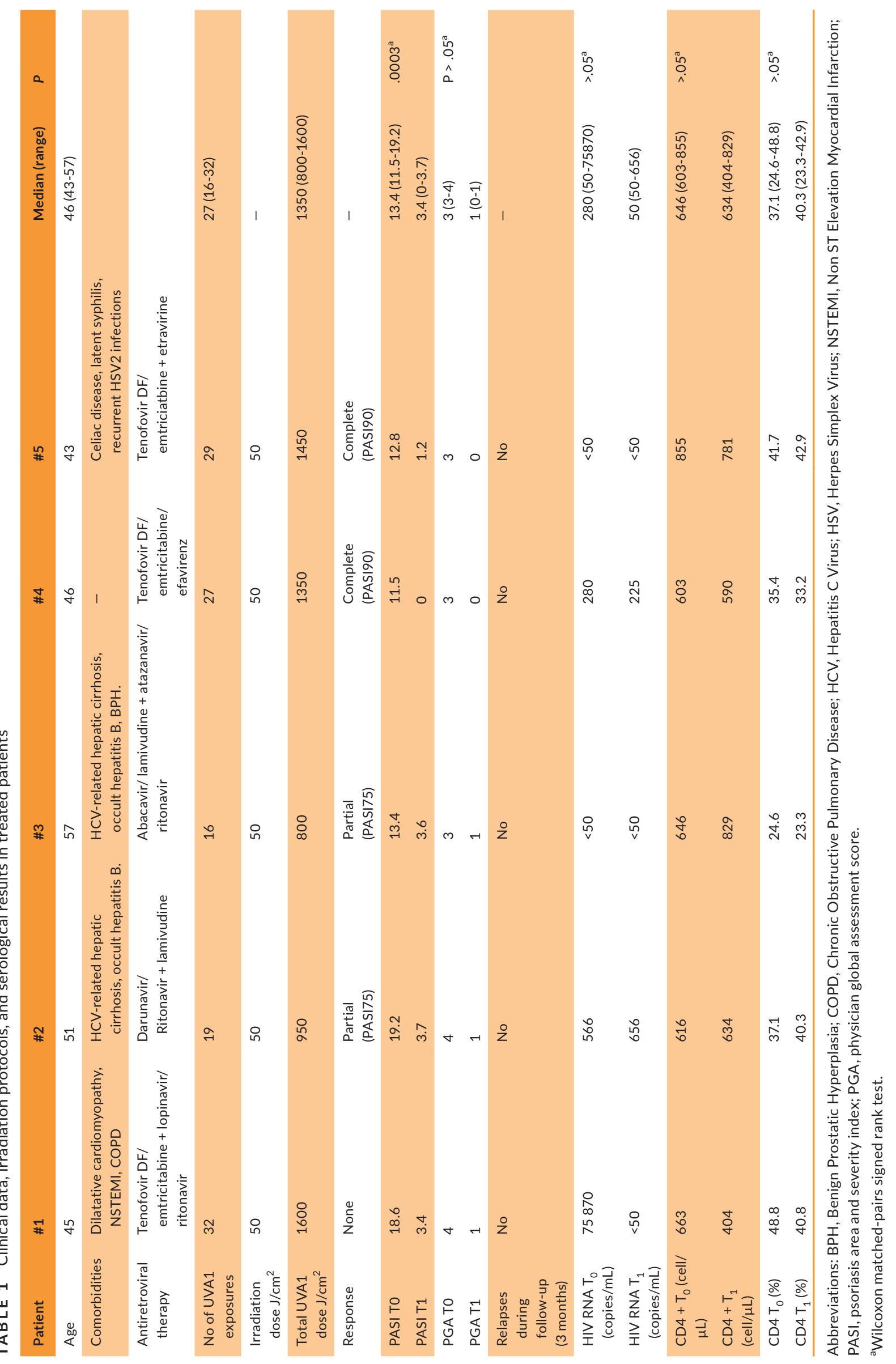




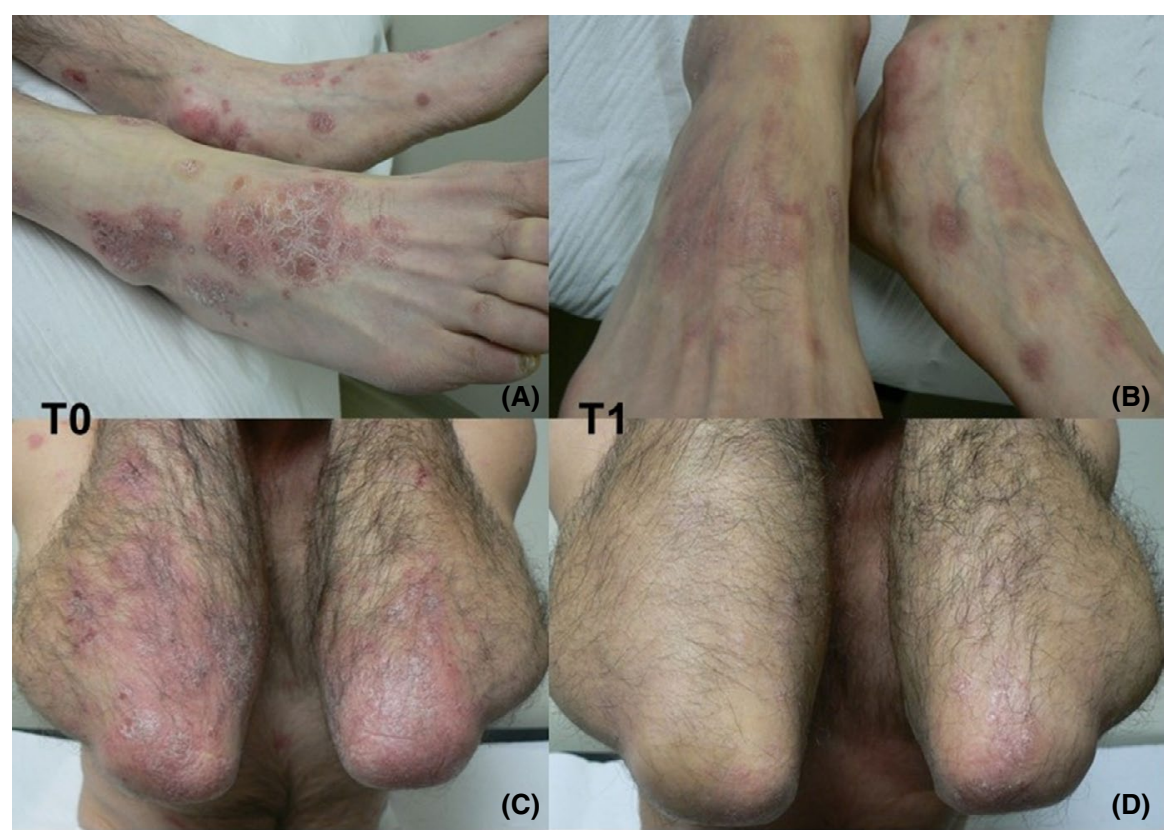

FIGURE 1 Psoriatic lesions before $(A, C)$ and after UVA-1 phototherapy (B,D) in a patient [Colour figure can be viewed at wileyonlinelibrary.com]

and UV-induced skin thickening and pigmentation may alter the effective radiation dose reaching HIV-infected skin cells.

With regard to UVA1 wavelength, the inhibiting effect on TNF $\alpha$ and INT $\gamma$ must be considered as these mediators have an inducing effect on HIV and immune cells infection. ${ }^{3}$ Furthermore, UVA-1 radiation can cause both early and late apoptosis. ${ }^{3}$ This dual effect might create some concern about an aggravating effect on the immunosuppressed state in HIV-infected patients but instead, it could be read as a way to eliminate infected T-cell in the skin. Obviously, we must remember that any kind of phototherapy does not induce a systemic immunosuppression, and this put us far from considering UVA1 phototherapy as a treatment option for people living with HIV.

In our small case series, UVA-1 phototherapy proved to be a safe and effective treatment option in psoriatic HIV-positive patients. Future results on larger case series will enable us to compare the efficacy of different types of irradiation sources and to better outline skin cytokines changes induced by phototherapy in this particular set of patients.

\section{CONFLICT OF INTEREST}

None to declare.

\section{REFERENCES}

1. Menon K, Van Voorhes AS, Bebo BF, et al. Psoriasis in patients with HIV infection: from the medical board of the National Psoriasis Foundation. J Am Acad Dermatol. 2010;62:291-299.

2. Akaraphanth R, Lim HWHIV. UV and immunosuppression. Photodermatol Photoimmunol Photomed. 1999;15:28-31.
3. Narumol S, Pattanaprichakul P, Charoenpipatsin N, et al. The efficacy of UVA1 phototherapy in psoriasis: clinical and histological aspects. Photoderm Photoimmunol Photomed. 2020;36:21-28.

4. Zmudzka BZ, Beer JZ. Activation of human immunodeficiency virus by ultraviolet radiation. Photochem Photobiol. 1990;52:1153-1162.

$$
\begin{array}{r}
\text { Mariachiara Arisi }{ }^{1} \text { iD } \\
\text { Alessandra Gelmetti }{ }^{1} \\
\text { Emanuele Focà }{ }^{2} \\
\text { Mariateresa Rossi }{ }^{1} \\
\text { Chiara Rovati }{ }^{1} \\
\text { Piergiacomo Calzavara-Pinton }{ }^{1} \\
\text { Marina Venturini }{ }^{1} \\
{ }^{1} \text { Dermatology Unit, ASST Spedali Civili, University of Brescia, } \\
\text { Brescia, Italy } \\
\text { Department of Infectious and Tropical Diseases, ASST Spedali } \\
\text { Civili , University of Brescia, Brescia, Italy } \\
\text { Correspondence } \\
\text { University of Brescia, ASST Spedali Civili of Brescia, Piazzale } \\
\text { Spedali Civili 1, 25123 Brescia, Italy. } \\
\text { Email: mariachiara.arisi@gmail.com }
\end{array}
$$

\section{Correspondence}

\section{ORCID}

Mariachiara Arisi iD https://orcid.org/0000-0001-6307-6174 